\title{
Modelo del transformador monofásico en el dominio de la frecuencia
}

\section{Single phase transformer model in the frequency domain}

ESCAMILLA-SÁNCHEZ, Juan Carlosi๋*, MORALES-AGUILAR, Eric, CANDIA-GARCÍA Filiberto, MAR, José Luis y MARTINEZ, Daniel F.

\author{
Benemérita Universidad Autónoma de Puebla \\ Universidad de Colima \\ ID $1^{\text {er }}$ Autor: Juan Carlos, Escamilla-Sánchez / CVU CONACYT ID: 230175 \\ ID $1^{\mathrm{er}}$ Coautor: Eric, Morales-Aguilar / CVU CONACYT ID: 225102 \\ ID $2^{\text {do }}$ Coautor: Filiberto, Candia-García / CVU CONACYT ID: 264231 \\ ID $3^{\text {er }}$ Coautor: José Luis, Mar \\ ID $4^{\text {to }}$ Coautor: Daniel F., Martínez
}

DOI: $10.35429 / J E A .2020 .23 .7 .1 .6$

Recibido: 25 de Abril, 2020; Aceptado: 20 de Mayo, 2020

\begin{abstract}
Resumen
En este artículo se presenta un modelo en el dominio de la frecuencia para el análisis de la transferencia de sobrevoltajes transitorios inducidos al secundario de un transformador monofásico, así como la distribución del voltaje transitorio a lo largo de ambos devanados. El transformador se modela como un elemento de parámetros distribuidos a partir de las ecuaciones del telegrafista en el dominio de la frecuencia. Para obtener la solución en el dominio del tiempo se emplea la Transformada Numérica de Laplace (TNL).
\end{abstract}

Modelado del transformador, Transformada Numérica de Laplace, transitorios electromagnético

\begin{abstract}
This article presents a model in the frequency domain for the analysis of the transient induced overvoltage transfer to the secondary of a single-phase transformer, as well as the distribution of the transient voltage along both windings. The transformer is modeled as an element of distributed parameters from the telegraphist's equations in the frequency domain. The Laplace Numerical Transform (TNL) is used to obtain the solution in the time domain.
\end{abstract}

Transformer modeling, Laplace Numerical Transform, electromagnetic transients Aplicaciones de la Ingeniería. 2020. 7-23: 1-6.

\footnotetext{
* Correspondencia del Autor (Correo Electrónico: escamilla_14@hotmail.com)

$\dagger$ Investigador contribuyendo como primer autor.
} 


\section{Introducción}

El estudio y comportamiento de los sobrevoltajes transitorios en el transformador han sido tópicos de gran interés e importancia en el área de ingeniería de potencia (Al-fuhaid, 2001), (Saied \& Al-fuhaid, 1985), (Saied \& Al-fuhaid, 2004), (Liang, Sun, Zhang, \& Cui, 2006), (Shibuya, Fijita, \& Hosokawa, 1997), (Dang \& Ridriguez, 1999), (Honorati \& Santini, 1990). Para el análisis de transitorios de altas frecuencias, generados tanto por descargas atmosféricas como por maniobras o fallas, se emplean modelos denominados internos, los cuales describen principalmente la distribución del potencial y la propagación en el devanado primario. Para ello, se emplean tanto representaciones de parámetros concentrados como de parámetros distribuidos.

Entre diversas técnicas de análisis, la teoría de la línea multiconductora en el dominio de la frecuencia se ha utilizado para el análisis de los sobrevoltajes transitorios en los devanados del transformador (Al-fuhaid, 2001), (Saied \& Al-fuhaid, Electromagnetic transient in a Line Transformer Cascade by a Numerical Laplace Transform Technique, 1985), (Shibuya, Fijita, \& Hosokawa, 1997). Por ejemplo, uno de los modelos basados en esta teoría describe el devanado completo del transformador mediante un modelo de parámetros distribuidos de la línea monofasica, y para el análisis a detalle del fenómeno utiliza un modelo de la línea multiconductora en las primeras vueltas (Shibuya, Fijita, \& Hosokawa, 1997). Otro modelo del transformador en el dominio de la frecuencia consiste en obtener una ecuación diferencial ordinaria (EDO) cuya solución es calculada por el método numérico de Runge Kutta y describe el comportamiento del devanado del transformador ante sobrevoltajes (Dang \& Ridriguez, 1999).

En este artículo se emplea un modelo del transformador para altas frecuencias (primordialmente descargas atmosféricas). A diferencia de los modelos usados generalmente, este modelo considera también la transferencia de sobrevoltajes transitorios al lado secundario. La naturaleza distribuida de los parámetros eléctricos del transformador se describe a partir de las ecuaciones del telegrafista comúnmente empleadas en el análisis de la línea de transmisión.
A partir de estas ecuaciones se obtiene un modelo de 2 puertos (nodal o matriz de admitancias) del transformador. Se incluye un ejemplo de aplicación en el cual se analiza la distribución de sobrevoltajes transitorios a lo largo de los devanados primario y secundario de un transformador de distribución, Para obtener la solución en el dominio del tiempo se emplea la Transformada Numérica de Laplace (TNL) (Vahidi, 2005).

\section{Modelo del transformador}

La representación circuital del transformador para una unidad de longitud se presenta en la Fig. 1 (Al-fuhaid, 2001). El modelo considera las siguientes aproximaciones: Se asumen devanados distribuidos uniformemente. Los parámetros del transformador se asumen constantes. La operación es considerada en la región lineal de la curva de magnetización. La inductancia mutua es considerada únicamente entre la vuelta del devanado primario que corresponde con la del secundario. Del circuito de la Fig. 1 se obtienen los cambios de voltaje en los devanados dados por.

$$
\left[\begin{array}{l}
\Delta V_{1}(x, s) \\
\Delta V_{2}(x, s)
\end{array}\right]=\left[\begin{array}{ll}
Z_{1}(s) \Delta x & Z_{m}(s) \Delta x \\
Z_{m}(s) \Delta x & Z_{2}(s) \Delta x
\end{array}\right]\left[\begin{array}{l}
\hat{I}_{1}(x, s) \\
\hat{I}_{2}(x, s)
\end{array}\right]
$$

Donde $\hat{I}_{1}(x, s)$ y $\hat{I}_{2}(x, s)$ son las corrientes fluyendo en $\mathrm{Z1}$ y $\mathrm{Z} 2$ respectivamente, $\mathrm{Zm}$ es la la impedencia de acoplamiento magnético entre debanados. Se tiene entonces las siguientes deficiniones:

$$
\begin{aligned}
& Z_{1}(s)=R_{1}+s L_{1} \\
& Z_{2}(s)=R_{2}+s L_{2} \\
& Z_{m}(s)=s L_{m}
\end{aligned}
$$

Expresando estas corrientes en función de la corriente total del devanado, dividiendo entre $\Delta \mathrm{x}$ y aplicamos el límite cuando $\Delta \mathrm{x} \rightarrow 0$, se obtiene:

$$
\left[\begin{array}{c}
\frac{d V_{1}(x, s)}{d x} \\
\frac{d V_{2}(x, s)}{d x}
\end{array}\right]=\frac{1}{D(s)}\left[\begin{array}{cc}
Z_{1}+Z_{1} Y_{2} Z_{2}-Z_{m}^{2} Y_{2} & Z_{m} \\
Z_{m} & Z_{2}+Z_{2} Y_{1} Z_{1}-Z_{m}^{2} Y_{1}
\end{array}\right]\left[\begin{array}{c}
I_{1}(x, s) \\
I_{2}(x, s)
\end{array}\right]
$$




$$
\begin{aligned}
& D(s)=1+Z_{1} Y_{1}+Z_{2} Y_{2}+Z_{1} Z_{2} Y_{1} Y_{2}-Z_{m}^{1} Y_{1} Y_{2} \\
& Y_{1}(s)=s C_{1}+1 / R_{c 1} \\
& Y_{2}(s)=s C_{2}
\end{aligned}
$$

Escribiendo la variación de corrientes respecto a $\Delta \mathrm{x}$ del devanado primario $\mathrm{y}$ del secundario, dividiendo entre $\Delta \mathrm{x}$ y aplicando los límites cuando $\Delta \mathrm{x} \rightarrow 0$ se tiene la siguiente ecuación:

$\left[\begin{array}{l}\frac{d I_{1}(x, s)}{d x} \\ \frac{d I_{2}(x, s)}{d x}\end{array}\right]=\left[\begin{array}{cc}Y_{g 1}(s)+Y_{m}(s) & -Y_{m} \\ -Y_{m} & Y_{g 2}(s)+Y_{m}(s)\end{array}\right]\left[\begin{array}{l}V_{1}(x, s) \\ V_{2}(x, s)\end{array}\right]$

Donde:

$Y_{g 1}(s)=s C_{g 1}, Y_{m}(s)=s C_{m}$ y $Y_{g 2}(s)=s C_{g 2}$

$(6 a b c)$

Las ecuaciones (3) y (5) en forma compacta se pueden escribir como.

$\left[\begin{array}{c}\frac{d \mathbf{V}(x, s)}{d x} \\ \frac{d \mathbf{I}(x, s)}{d x}\end{array}\right]=\left[\begin{array}{cc}\mathbf{0} & \mathbf{Z}(s) \\ \mathbf{Y}(s) & \mathbf{0}\end{array}\right]\left[\begin{array}{l}\mathbf{V}(x, s) \\ \mathbf{I}(x, s)\end{array}\right]$

$V(x, s)$ e $I(x, s)$ son los vectores de voltaje y de corriente en el dominio de Laplace en el punto x del devanado, mientras que $Z(s)$ y $Y(s)$ son las matrices de impedancias y admitancias descritas en las ecuaciones (3) y (5). Las ecuaciones acopladas de primer orden definidas en (7) pueden convertirse en ecuaciones desacopladas de segundo orden:

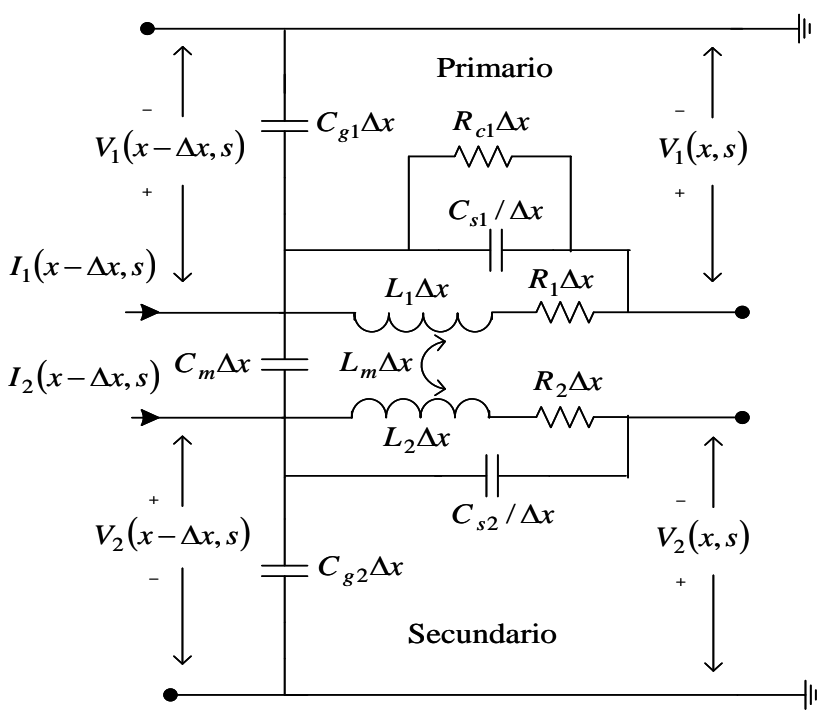

Figura 1 Representación para un diferencial de longitud del transformador $\left[\begin{array}{c}\frac{d^{2} \mathbf{V}(x, s)}{d x^{2}} \\ \frac{d^{2} \mathbf{I}(x, s)}{d x^{2}}\end{array}\right]=\left[\begin{array}{cc}\mathbf{Z}(s) \mathbf{Y}(s) & \mathbf{0} \\ \mathbf{0} & \mathbf{Z}(s) \mathbf{Y}(s)\end{array}\right]\left[\begin{array}{l}\mathbf{V}(x, s) \\ \mathbf{I}(x, s)\end{array}\right]$

La solución general del sistema definido en (8) está dada por:

$$
\begin{aligned}
& \mathbf{V}(x, s)=\exp (-\boldsymbol{\Psi} x) \mathbf{C}_{1}+\exp (\boldsymbol{\Psi} x) \mathbf{C}_{2} \\
& \mathbf{I}(x, s)=\mathbf{Y}_{0}\left[\exp (-\boldsymbol{\Psi} x) \mathbf{C}_{1}-\exp (\boldsymbol{\Psi} x) \mathbf{C}_{2}\right]
\end{aligned}
$$

Donde $\boldsymbol{\Psi} x$ es la matriz de constantes de propagación del devanado, la cual esta en función de los vectores y valores propios, definida como:

$\boldsymbol{\Psi} x=\boldsymbol{M} \sqrt{\lambda} \boldsymbol{M}^{-1}$

M y $\lambda$ son las matrices de vectores y valores propios del producto $\mathbf{Z}(s) \mathbf{Y}(s)$, respectivamente, y $\mathbf{Y}_{0}$ es la matriz de admitancias características del devanado, calculada como sigue:

$\mathbf{Y}_{0}=\mathbf{Z}(\mathrm{x}, \mathrm{s})^{-1} \boldsymbol{\Psi} x$

Aplicando las condiciones de frontera de Dirichlet, se conosdera $x=0$ y $x=L$ en (9a) y (9b), puede obtenerse el modelo de 2 puertos conocido como forma nodal o de matriz de admitancias (Fig. 2):

$\left[\begin{array}{l}\mathbf{I}(0, s) \\ \mathbf{I}(L, s)\end{array}\right]=\left[\begin{array}{cc}\mathbf{A} & -\mathbf{B} \\ -\mathbf{B} & \mathbf{A}\end{array}\right]\left[\begin{array}{l}\mathbf{V}(0, s) \\ \mathbf{V}(L, s)\end{array}\right]$

Donde:

$\mathbf{A}=\mathbf{Y}_{0} \operatorname{coth}(\boldsymbol{\Psi} x L)$

$\mathbf{B}=\mathbf{Y}_{0} \operatorname{csch}(\boldsymbol{\Psi} x L)$

A partir del modelo de 2 puertos se obtienen los voltajes en las terminales del transformador en el dominio de la frecuencia. Los valores respectivos en el dominio del tiempo se obtienen a partir del algoritmo de Transformada Numérica de Laplace, descrito en la siguiente sección. 


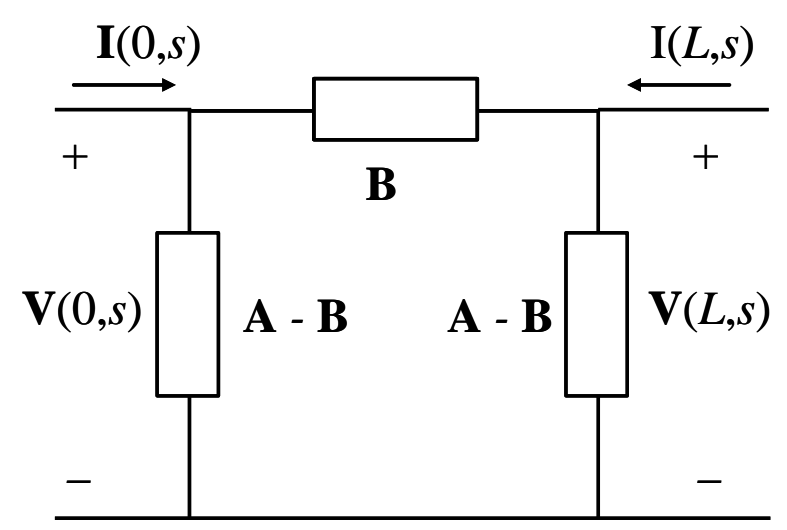

Figura 2 Modelo de 2 puertos del transformador

\section{Ejemplo de Aplicación}

Un transformador monofásico de 15 MVA, 34.5./13.8 KV $60 \mathrm{~Hz}$ es utilizado para mostrar la técnica descrita. Los parámetros de transformador por unidad de longitud son los siguientes:

$\begin{array}{ll}\text { Devanado Primario: } & \text { Devanado Secundario: } \\ \mathrm{R}_{1}=0.22 \Omega & \mathrm{R}_{2}=0.0366 \Omega \\ \mathrm{R}_{\mathrm{s} 1}=130 \mathrm{~K} \Omega, & \mathrm{L}_{\mathrm{s} 2}=1.18 \mathrm{mH} \\ \mathrm{L}_{\mathrm{s} 1}=7.35 \mathrm{mH} & \mathrm{C}_{\mathrm{g} 2}=27 \mathrm{nF} \\ \mathrm{C}_{\mathrm{g} 1}=9 \mathrm{nF} . & \end{array}$

Para ambos devanados se asume que

$$
\frac{\alpha^{2}}{l^{2}}=\frac{C_{g k}}{C_{s k}}=10
$$

La inductancia mutua es $\mathrm{L}_{\mathrm{m}}=2.8 \mathrm{mH}$ y la capacitancia mutua es $\mathrm{C}_{\mathrm{m}}=148 \mathrm{pF}$. En el lado del primario del transformador se suministra una señal de tipo escalón unitario. La longitud del devanado analizada es de 10 metros $(\mathrm{L}=10 \mathrm{~m})$, dividida en 4 segmentos, tal como se muestra en la Fig. 3. En dicha figura las seccions se clasifican como:

\section{A = Escalón suministrado al inicio del devanado primario}

$\mathrm{B}=$ Voltaje transitorio a 1/4 del devanado

$\mathrm{C}=$ Voltaje transitorio a 2/4 del devanado

$\mathrm{D}=$ Voltaje transitorio a $3 / 4$ del devanado

En la Fig. 4 se observan los sobrevoltajes transitorios presentes en los diferentes segmentos del devanado primario, mientras que en la Fig. 5 se presentan los sobrevoltajes transferidos al secundario.
En el instante de incidencia del impulso prácticamente sólo las capacitancias del circuito reaccionan al escalón suministrado. La distribución de potencial en dicho instante (distribución inicial) se observa en la Fig. 6, la cual se obtiene en un tiempo $\mathrm{t}=0.1 \mu \mathrm{s}$. En el devanado primario el comportamiento es exponencial decreciente, mientras que en el secundario es prácticamente cero, es decir, aún no hay transferencia a considerar. Conforme aumenta el tiempo los elementos inductivos del devanado se involucran en el fenómeno y se presenta el sobrevoltaje transitorio. En la Fig. 6 tambien muestra la distribución de potencial para un tiempo $\mathrm{t}=0.297 \mathrm{~ms}$ en el cual se alcanza el máximo sobrevoltaje. Se observa también que dicho máximo se presenta en aproximadamente el $30 \%$ de la longitud para ambos devanados (1.46 p.u. en el primario y 0.61 p.u. en el secundario, con los valores por unidad referidos al primario). Al final del evento transitorio los elementos resistivos gobiernan la respuesta del circuito, por lo que la transferencia de potencial se comporta de manera lineal. En la Fig. 7 se grafica el comportamiento de la distribución de potencial ante la variación de la magnitud de $\alpha$, considerando el tiempo en que se presenta el máximo sobrevoltaje en cada caso. Idealmente, con $\alpha \rightarrow 0$, el transitorio prácticamente no se presenta. El sobrevoltaje transitorio en ambos devanados se atenuará entonces reduciendo $\mathrm{C}_{\mathrm{gk}} \mathrm{O}$ nulificándola parcial o totalmente, o bien, incrementando la capacitancia serie Cs.

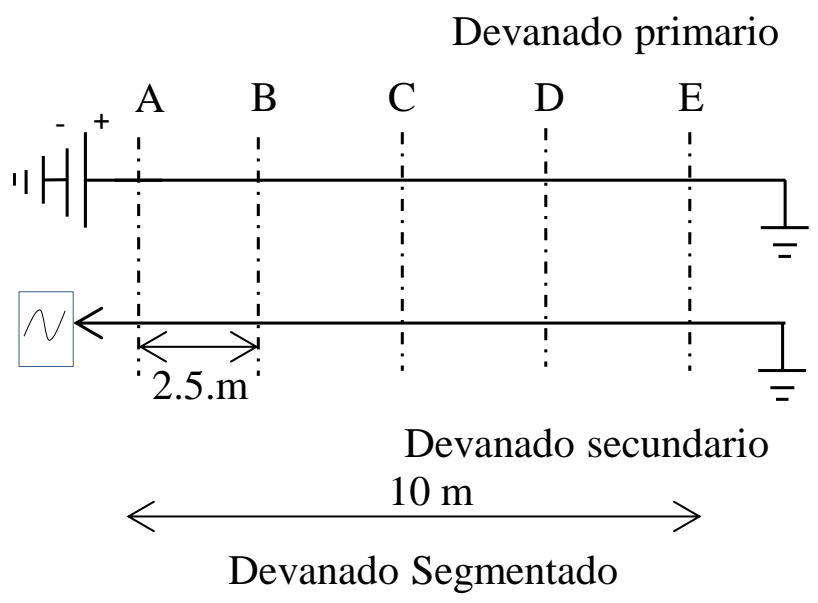

Figura 3 Diagrama para el ejemplo de aplicación 


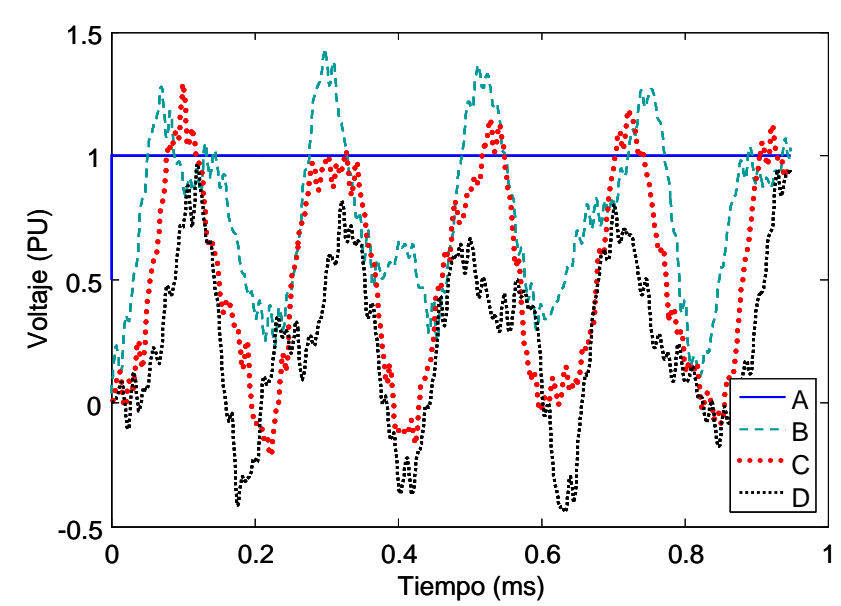

Figura 4 Voltaje transitorio a lo largo del devanado primario para $\alpha=10$

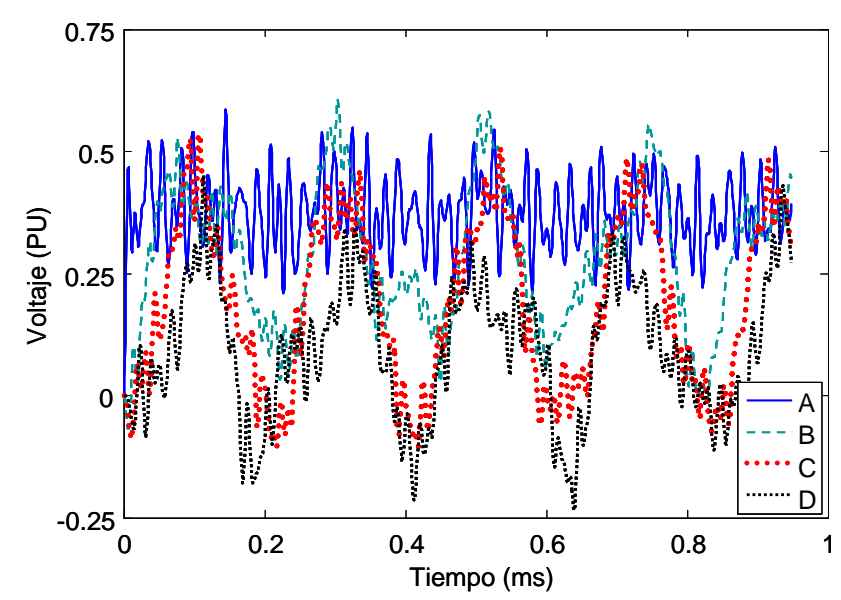

Figura 5 Voltaje transitorio a lo largo del devanado secundario
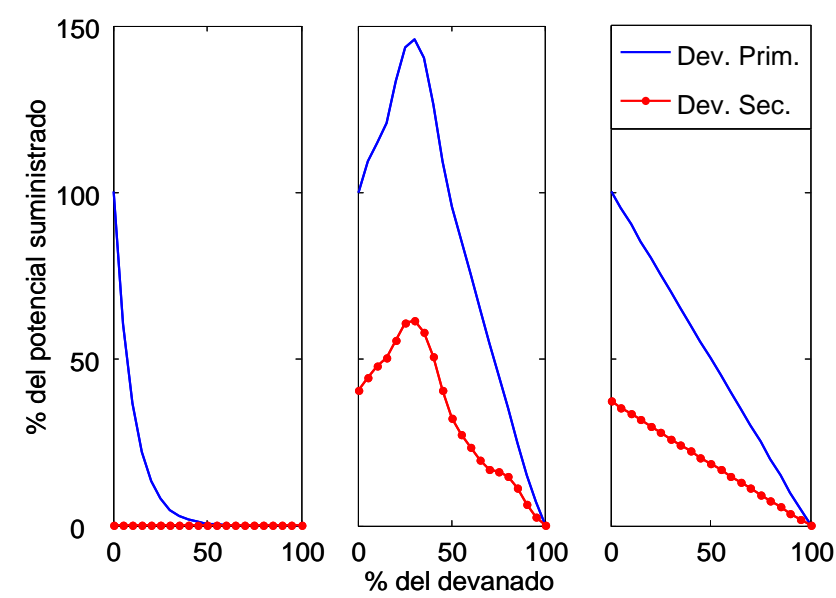

Figura 6 Distribución de Potencial
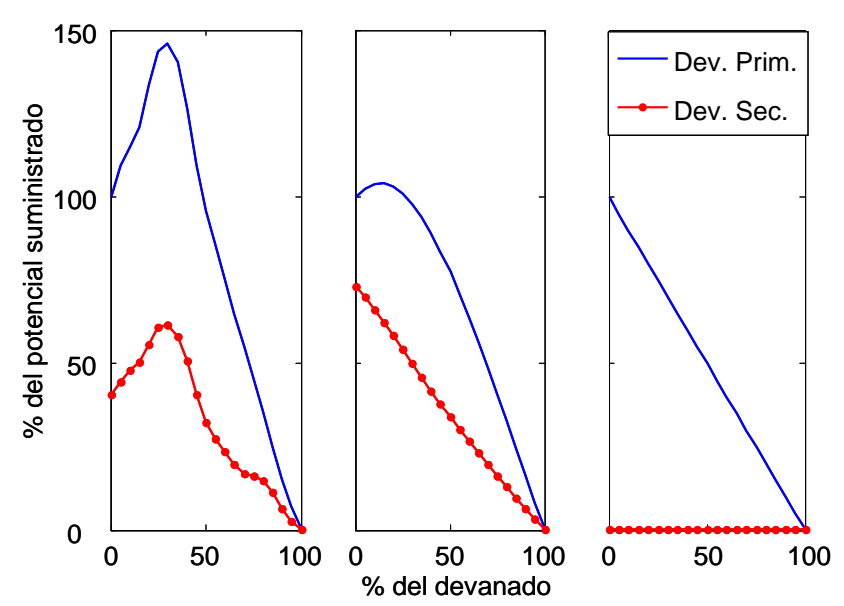

Figura 7 Distribución de Potencial (máximo) con diferentes valores de $\alpha$

\section{Agradecimientos}

Los autores agradecen a la Benemérita Universidad Autónoma de Puebla y a la Universidad de Colima campus Manzanillo por otorgar las facilidades para la presentación de este trabajo.

\section{Conclusiones}

En este trabajo se desarrolló un modelo en el dominio de la frecuencia para analizar el comportamiento de la propagación y transferencia de sobrevoltajes en los devanados de un transformador. El modelo se basa en la solución de las ecuaciones de telegrafista en el dominio de la frecuencia, utilizando parámetros del transformador obtenidos de su geometría. Además de las curvas de distribución de potencial en el devanado primario obtenidas por modelos internos típicos, mediante este modelo es posible obtener también la distribución de potencial transferido al secundario. La forma en la cual una onda de alta frecuencia se transfiere al secundario del transformador es de alta importancia práctica. Podría determinar, por ejemplo, la descarga que aparece en un bus de generación cuando una onda incide en las terminales de alto voltaje de un transformador elevador.

\section{Referencias}

Al-fuhaid, A. S. (2001). Frequency Characteristics of Single Phase Two Winding Transformer Using Distributed Parameter Modeling. IEEE Trans. Power Delivery, 637642. 
Dang, H., \& Ridriguez , H. (1999). Behavior of transformer windings under voltages. High Voltage Engineering Symposium (págs. 12871290). IEE Conference Publication.

Honorati, O., \& Santini, E. (1990). New approach to the analysis of impulse voltage distribution in transformer windings. IEE, 283290.

Liang, G., Sun, H., Zhang, X., \& Cui, X. (2006). Modeling of Transformer Windings Under Very Fast Transient Overvoltages. IEEE Trans. Electromagnetic Compatibility, 1 - 7.

Saied , M., \& Al-fuhaid, A. S. (1985). Electromagnetic transient in a Line Transformer Cascade by a Numerical Laplace Transform Technique. IEEE Trans. On Power Apparatus and System, 2901-2909.

Saied, M., \& Al-fuhaid, A. (2004). Frequency Response of Two-Winding Transformers Obtained by a Distributed -Parameter s-Domain Method. Electric Power Components an systems, 755-766.

Shibuya, Y., Fijita, S., \& Hosokawa, N. (1997). Analysis of very Fast Transient overvoltages in transformer windings. IEE Proc.-Gener. Transm. Distribution, 1 - 7.

Vahidi, B. (2005). Using Pspice in Teaching Impulse Voltage Testing of Power Transformer to Senior Undergraduate Students. IEEE Transaction on Education, 1 - 7. 